\section{Has the PROPHECY of AR-V7 Been Fulfilled?}

\section{TO THE EDITOR:}

There is an unmet clinical need for predictive biomarkers in metastatic castration-resistant prostate cancer (mCRPC) resulting in unnecessary adverse effects and costs. Conversely, there are several prognostic biomarkers, such as circulating tumor cell (CTC) counts, lactate dehydrogenase, and prostate-specific antigen concentrations that have limited clinical utility for guiding treatment selection. Armstrong et $\mathrm{al}^{1}$ recently published the first data from the PROPHECY trial (ClinicalTrials.gov identifier: NCT02269982; Prospective Circulating Prostate Cancer Predictors in Higher Risk mCRPC Study), a multicenter cohort study that collected liquid biopsies with the primary goal of validating the prognostic value of the androgen receptor splice variant 7 (AR-V7) in men with $\mathrm{mCRPC}$ treated with AR signaling inhibitors (either abiraterone or enzalutamide). We applaud the authors for their great effort to perform a validation trial. However, we have concerns about their claims and statements regarding how the analysis was performed, the selected patient cohort, and the lack of prospective randomized clinical trials (RCTs) supporting the treatment-predictive capability of AR-V7.

The PROPHECY trial report discusses two different CTC-dependent assays for detecting AR-V7: the Johns Hopkins University (JHC, Baltimore, MD) modified-AdnaTest CTC AR-V7 mRNA assay and the Epic Sciences (Epic; San Diego, CA) CTC nuclearspecific AR-V7 protein assay. The number of CTCs detected in $7.5 \mathrm{~mL}$ of blood, assessed by the CELLSEARCH system (Menarini Silicon Biosystems; Bryn Athyn, PA) is a highly prognostic continuous variable. ${ }^{2}$ Although we understand that CTCs were incorporated as the FDA-cleared dichotomous variable with a cutoff of five CTCs, this limits the variance explained by the CTC counts. An additional analysis using CELLSEARCH CTCs as a continuous variable would be of great interest to the community to fully understand whether AR-V7 harbors independent prognostic information or if simpler and more widely accessible CELLSEARCH CTC enumeration could instead be applied for patient prognostication. In addition, other prognostic variables were not individually assessed in the multivariable analysis; instead, the Halabi risk score was used. The Halabi risk score was calibrated to predict overall survival for patients receiving first-line chemotherapy. ${ }^{3}$ A supplementary analysis would have been of interest in which the prognostic variables behind the Halabi score were applied directly in a multivariable analysis to infer potential correlations and interaction effects.

Furthermore, the patients included in the PROPHECY trial were preselected to have two or more poorprognosis clinical factors, with half the cohort having six or more. Importantly, $36 \%$ of patients received previous treatment with an AR signaling inhibitor, which is a known poor-prognosis factor in the context of enzalutamide or abiraterone, and it increases the likelihood of detectable treatment-induced expression of splice variants, including AR-V7. ${ }^{4}$ In addition, PROPHECY patients with CTCs but without detectable AR-V7 expression did not progress faster than CTC-negative patients, regardless of whether the AdnaTest CTC AR-V7 mRNA assay or the CTC nuclear-specific AR-V7 protein assay was used. This result was unexpected because an increased tumor burden in itself is strongly associated with progressionfree survival, regardless of treatment. ${ }^{5}$ Consequently, these data are relevant only for preselected poorprognosis patients and cannot be extrapolated to an all-comer setting.

Finally, the PROPHECY trial was designed to validate the prognostic value of AR-V7. However, in their discussion, the authors speculate that AR-V7 is now ready to be applied as a predictive biomarker for stratification of patients with MCRPC to either taxanes or AR signaling inhibitors on the basis of their previous work. 6,7 However, the studies reported in those articles did not prospectively randomly assign patients on the basis of their AR-V7 status and were themselves controversial. ${ }^{8}$ Until formally tested in an RCT, it is not known whether the response rate of patients with AR-V7 is higher with taxanes compared with AR signaling inhibitors. Because the authors of the PROPHECY trial are distinguished leaders in their field, their opinions have considerable impact, and we are concerned that the general conclusion resulting from their study has been that AR-V7 is now ready for prime time as a predictive biomarker. ${ }^{9}$

Going forward, we encourage the authors of the PROPHECY trial to conduct an RCT to provide conclusive evidence regarding whether AR-V7 can be applied as a predictive biomarker or not. Since the initial association of AR-V7 with AR signaling inhibitors in 2014, new knowledge has emerged. We now know that TP53 mutation carriers respond poorly ${ }^{4,10}$ and that the $A R$ locus is hosting a plethora of genomic alterations and noncanonical transcripts. ${ }^{4}$ 
Comprehensive liquid biopsy profiling, beyond any single biomarker, is therefore going to be needed to improve stratification of patients with mCRPC. To address this essential and unmet need, several prospective multiarm clinical trials incorporating liquid biopsy profiling such as the IND.234 (ClinicalTrials.gov identifier: NCT03385655; Prostate Cancer Biomarker Enrichment and Treatment Selection) and ProBio (ClinicalTrials.gov identifier: NCT03903835; ProBio: A Biomarker Driven Study in Patients With Metastatic Castrate Resistant Prostate Cancer) have been initiated that will eventually result in the identification of single biomarkers or biomarker signatures (eg, DNA repair deficiency) that are predictive for personalized treatment selection.

\section{Bram De Laere, PhD}

Karolinska Institutet, Stockholm, Sweden

Piet Ost, MD, PhD

Ghent University Hospital, Ghent, Belgium

Henrik Grönberg, MD, PhD and Johan Lindberg, PhD Karolinska Institutet, Stockholm, Sweden

AUTHORS' DISCLOSURES OF POTENTIAL CONFLICTS OF INTEREST AND DATA AVAILABILITY STATEMENT

Disclosures provided by the authors and data availability statement (if applicable) are available with this article at DOI https://doi.org/10.1200/ JCO.19.01015

\section{REFERENCES}

1. Armstrong AJ, Halabi S, Luo J, et al: Prospective multicenter validation of androgen receptor splice variant 7 and hormone therapy resistance in high-risk castration-resistant prostate cancer: The PROPHECY study. J Clin Oncol 37:1120-1129, 2019

2. Scher HI, Jia X, de Bono JS, et al: Circulating tumour cells as prognostic markers in progressive, castration-resistant prostate cancer: A reanalysis of IMMC38 trial data. Lancet Oncol 10:233-239, 2009

3. Halabi S, Lin CY, Kelly WK, et al: Updated prognostic model for predicting overall survival in first-line chemotherapy for patients with metastatic castration-resistant prostate cancer. J Clin Oncol 32:671-677, 2014

4. De Laere B, Oeyen S, Mayrhofer M, et al: TP53 outperforms other androgen receptor biomarkers to predict abiraterone or enzalutamide outcome in metastatic castration-resistant prostate cancer. Clin Cancer Res 25: 1766-1773, 2019

5. Bozic I, Reiter JG, Allen B, et al: Evolutionary dynamics of cancer in response to targeted combination therapy. eLife 2:e00747, 2013

6. Antonarakis ES, Lu C, Luber B, et al: Androgen receptor splice variant 7 and efficacy of taxane chemotherapy in patients with metastatic castrationresistant prostate cancer. JAMA Oncol 1:582-591, 2015

7. Scher HI, Graf RP, Schreiber NA, et al: Assessment of the validity of nuclear-localized androgen receptor splice variant 7 in circulating tumor cells as a predictive biomarker for castration-resistant prostate cancer. JAMA Oncol 4:1179-1186, 2018

8. Plymate SR, Sharp A, de Bono JS: Nuclear circulating tumor cell androgen receptor variant 7 in castration-resistant prostate cancer: The devil is in the detail. JAMA Oncol 4:1187-1188, 2018

9. OncLive: CTC AR-V7 validated as predictive marker of resistance to AR-directed therapy in mCRPC. https://uww.onclive.com/web-exclusives/ctc-arv7-validatedas-predictive-marker-of-resistance-to-ardirected-therapy-in-mcrpc

10. Annala M, Vandekerkhove $\mathrm{G}$, Khalaf $\mathrm{D}$, et al: Circulating tumor DNA genomics correlate with resistance to abiraterone and enzalutamide in prostate cancer. Cancer Discov 8:444-457, 2018

DOI: https://doi.org/10.1200/JC0.19.01015; Published at jco.org on July 2, 2019. 
AUTHORS' DISCLOSURES OF POTENTIAL CONFLICTS OF INTEREST AND DATA AVAILABILITY STATEMENT

\section{Has the PROPHECY of AR-V7 Been Fulfilled?}

The following represents disclosure information provided by authors of this manuscript. All relationships are considered compensated. Relationships are self-held unless noted. I = Immediate Family Member, Inst = My Institution. Relationships may not relate to the subject matter of this manuscript. For more information about ASCO's conflict of interest policy, please refer to www.asco.org/rwc or ascopubs.org/jco/site/ifc.

Bram De Laere

Patents, Royalties, Other Intellectual Property: Patent W02017207702

pending for androgen receptor splice variants and androgen deprivation therapy.

Piet Ost

Consulting or Advisory Role: Ferring Pharmaceuticals (Inst), Janssen-Cilag (Inst), Bristol-Myers Squibb (Inst), Bayer (Inst)

Research Funding: MSD (Inst), Varian Medical Systems (Inst)

Travel, Accommodations, Expenses: Ferring Pharmaceuticals

\section{Henrik Grönberg}

Honoraria: Astellas Pharma, Janssen-Cilag, Bristol-Myers Squibb

Patents, Royalties, Other Intellectual Property: Patents pending for: methods and compositions for correlating genetic markers with risk of aggressive prostate cancer, method for indicating the presence or absence of prostate cancer, prognostic method for individuals with prostate cancer, method for indicating a presence or absence of aggressive prostate cancer, methods and compositions for correlating genetic markers with risk of aggressive prostate cancer.

No other potential conflicts of interest were reported. 